\title{
Diversidade de Arctiinae (Lepidoptera, Arctiidae) em área alterada em Altamira, Amazônia Oriental, Pará, Brasil
}

\author{
José Augusto TESTON ${ }^{1} \&$ Márcia Cristina DELFINA ${ }^{2}$
}

\begin{abstract}
RESUMO
Este trabalho avaliou a fauna de Arctiinae em Altamira, Pará, numa área com forte ação antrópica, por meio de armadilhas luminosas, com capturas de duas noites a cada fase de lua nova por mês, no período de dezembro de 2007 a novembro de 2008. Na avaliação foram utilizados os parâmetros: riqueza, abundância, constância, índices de diversidade e uniformidade de Shannon (H' e E') e Brillouin (H e E), dominância de Berger-Parker (BP). As estimativas de riqueza foram feitas através dos procedimentos não paramétricos, "Bootstrap", "Chao 1", "Chao 2", "Jackknife 1", "Jackknife 2" e "Michaelis-Mentem". Foram capturados 420 espécimes pertencentes a 64 táxons de Arctiinae, sendo 19 espécies registradas pela primeira vez no estado do Pará. Os valores dos parâmetros analisados para todo o período foram: $\mathrm{H}^{\prime}=4,69, \mathrm{E}=0,781, \mathrm{H}=4,37, \mathrm{E}=0,732 \mathrm{e}$ $\mathrm{BP}=0,183$. Durante o período menos chuvoso (junho-novembro) foram encontrados os valores mais significativos para todos os parâmetros analisados. No período mais chuvoso (dezembro-maio) foram encontrados os mais significativos percentuais de similaridade entre os meses. Os estimadores previram o encontro de mais espécies, entre 18,7\% a 60,9\%.
\end{abstract}

PALAVRAS-CHAVE: Biodiversidade, Mariposas, Noctuoidea, Região Neotropical

\section{Diversity of Arctiinae (Lepidoptera, Arctiidae) in disturbed area at Altamira, Eastern Amazon, Pará, Brazil}

\section{ABSTRACT}

This work evaluates the Arctiinae fauna in Altamira, Pará, in an area with strong human pressure, using light-traps during two nights at each stage of the new moon each month, from December 2007 to November 2008. The evaluation parameters were: richness, abundance, constancy, diversity index and evenness (Shannon H 'and E') and Brillouin ( $\mathrm{H}$ and E), dominance of Berger-Parker (BP). The richness estimatives were made by means of non-parametric procedures, Bootstrap, Chao 1, Chao 2, Jackknife 1, Jackknife 2 and Michaelis-Mentem. We captured 420 specimens belonging to 64 species of Arctiinae, and 19 species recorded for the first time in the state of Pará. The values of the parameters analyzed for the total period were: $H^{\prime}=4.69 ; E^{\prime}=0.781 ; H=4.37 ; E=0.732$ and $B P=0.183$. During the less rainy season (June-November) we found the most significant values for all parameters. In the rain season (December to May) we found the most significant percentage of similarity. Richness estimators predicted the gathering of more than $18.7 \%$ to $60.9 \%$ of species.

KEYWORDS: Biodiversity, Moths, Neotropical region, Noctuoidea,

1 Universidade Federal do Oeste do Pará - UFOPA, Programa de Ciências Biológicas, Av. Marechal Rondon, s/n - Bairro Caranazal - 68040-070 - Santarém, PA. E-mail: jateston@ ufpa.br

2 Universidade Federal do Pará - UFPA, Faculdade de Ciências Biológicas, Rua Coronel José Porfírio, 2515 - Bairro São Sebastião - 68372-040 - Altamira, PA. 


\section{INTRODUÇÃO}

A Floresta Amazônica possui níveis de biodiversidade muito pouco conhecidos, apesar de ser um dos biomas brasileiros mais intensamente estudados nas últimas décadas em termos de seu funcionamento ecológico. Estima-se que mais de 70\% das espécies ainda não estejam descritas e, considerando-se o ritmo atual de estudos de inventário, tal situação permanecerá assim, por muito tempo (Salati et al., 2006).

Altamira sofre uma considerável mudança na sua cobertura florestal original, devido à ação antrópica presente na zona de influência da rodovia Transamazônica (BR-230) e suas transversais, cuja ocupação humana tem sido fortemente induzida por projetos de colonização agrária nos últimos 30 anos (Salomão et al., 2007).

$\mathrm{Na}$ Amazônia Oriental, principalmente entre os rios Xingu e Iriri, que engloba parte dos municípios de São Félix do Xingu e Altamira, no estado do Pará, de acordo com estimativas feitas com os dados do Projeto de Estimativa do Desflorestamento Bruto da Amazônia (PRODES), o desmatamento intensificouse a partir do final da década de 1990 , cuja área desmatada acumulada aumentou de $347 \mathrm{~km} 2 \mathrm{em} 1997$ para $2.955 \mathrm{~km}^{2}$ em 2004 (Escada et al., 2005).

As principais unidades de paisagens, nesta regiáo, são: florestas ombrófilas ou úmidas, podendo ser abertas ou densas; vegetação aluvial, diretamente influenciada pelo sistema hidrológico do rio Xingu; florestas secundárias recentes e antigas, áreas de capoeiras abandonadas pela agricultura familiar e uso agropecuário, que agrupa áreas de culturas de ciclos curtos e longos e, pastagens. Em média, cerca de 60\% da cobertura vegetal original já foram convertidos para uso agropecuário (Salomão et al., 2007).

A destruição da floresta tropical e a extinção de espécies exigem a ampliação de estratégias de conservaçáo, uso sustentado e recuperaçâo de fragmentos remanescentes, bem como de áreas degradadas (Espírito-Santo et al., 2002).

Existe uma vasta literatura sobre efeitos de perturbação de floresta e fragmentação em comunidades de artrópodes tropicais (Hilt \& Fiedler, 2006). Considerando que mais da metade das espécies descritas no mundo são insetos, este grupo é de fundamental importância para o entendimento dos padróes da biodiversidade (Ferro \& Diniz, 2007).

Os lepidópteros, uma das principais ordens de insetos quanto à riqueza de espécies, sáo adequadas para estudos sobre a biodiversidade (Intachat \& Woiwod, 1999; Landau et al., 1999; Kitching et al., 2000; Hawes et al., 2009). Os arctiídeos estão entre as principais famílias de lepidópteros noturnos utilizados em monitoramento de ecossistemas naturais (Hilty \& Merenlender, 2000; Freitas et al., 2006) e alguns estudos mais recentes vêm ressaltando esta importância (Hilt
\& Fiedler, 2005; 2006; Brehm \& Axmacher, 2006; Brehm, 2007; Hilt, et al., 2007; Conner, 2009; Hawes et al., 2009).

Na região Neotropical são conhecidas 4.761 espécies de Arctiinae (Heppner, 1991). Segundo Brown Jr \& Freitas (1999), há uma estimativa de duas mil espécies para o Brasil. Já, para o estado do Pará, após ampla revisão bibliográfica, registramos 466 espécies.

No Brasil, a fauna de lepidópteros noturnos tem sido amplamente estudada com armadilhas luminosas, especialmente na determinação de índices faunísticos e distribuição desses organismos (Teston et al., 2006).

Este trabalho analisou a fauna de Arctiinae noturnos, quanto a sua composição, riqueza, abundância e diversidade durante os períodos mais e menos chuvosos, entre dezembro de 2007 a novembro de 2008, numa área alterada em Altamira, Amazônia Oriental brasileira, Pará.

\section{MATERIAL E MÉTODOS}

As atividades de coletas, realizadas nos períodos mais chuvoso e menos chuvoso, foram mensais, durante duas noites a cada período de lua nova, de dezembro de 2007 a novembro de 2008, totalizando 24 amostragens, no sítio Vista Alegre (0307'22”S; 52¹5'17'W, altitude $130 \mathrm{~m}$ ), localizado no município de Altamira, Pará. A área de estudo apresenta forte açáo antrópica e é constituída por pastagens e pomar com aproximadamente 25 anos de uso.

$\mathrm{Na}$ regiáo do estudo o período mais chuvoso estende-se de dezembro a maio, o menos chuvoso de junho a novembro (Moraes et al., 2005). Segundo as informaçôes obtidas na Estação de Meteorologia de Altamira, junto ao $2^{\circ}$ Distrito de Meteorologia (DISME) do Instituto Nacional de Meteorologia (INMET), durante o período de amostragem, foi apurada uma temperatura média de $27,2^{\circ} \mathrm{C}$ e pluviosidade total de $2.462,1$ $\mathrm{mm}$, sendo no período mais chuvoso $26,4^{\circ} \mathrm{C}$ e $2.001,1 \mathrm{~mm}$ e, no menos chuvoso $28^{\circ} \mathrm{C}$ e $461 \mathrm{~mm}$ de temperatura média e pluviosidade, respectivamente.

Para a captura dos arctiíneos foi utilizada armadilha luminosa EMATER/RS, modelo "Santa Rosa" (Kober, 1982) adaptada, possuindo um tronco de cone plástico com maior diâmetro de $32 \mathrm{~cm}$ e menor de $16 \mathrm{~cm}$, ao qual está acoplado um balde plástico com capacidade de 3,5 litros, onde são colocados 2 litros de álcool $92^{\circ}$ GL e, equipada com lâmpada fluorescente ultravioleta F15 T8 BLB, cuja luz possui comprimento de onda que varia de 290 a 450 e pico ao redor de 340 nanômetros. A armadilha foi instalada a uma altura de 2 metros do solo, acionada por bateria $12 \mathrm{~V}$, ao anoitecer $(18 \mathrm{~h})$ e desligada na manhã seguinte $(6 \mathrm{~h})$.

A triagem, identificação e contagem dos arctíneos, foi realizada no Laboratório Integrado de Biologia e Educação Ambiental (LIBEA) da Faculdade de Ciências Biológicas do Campus Universitário de Altamira da Universidade Federal do 
Pará (UFPA). O processo de identificação, o posicionamento sistemático adotado e a distribuiçáo das espécies dentro dos gêneros e tribos seguem Teston \& Corseuil (2002; 2003a; b; 2004). Uma parte dos espécimes coletados foi preparada a seco, em alfinetes entomológicos e a outra parte está conservada em envelopes entomológicos. O material testemunha (coleção de referência) encontra-se depositado na Coleção Entomológica do Museu de Zoologia do Programa de Ciências Biológicas da Universidade Federal do Oeste do Pará.

$\mathrm{Na}$ análise dos dados, calculou-se a constância das espécies em cada mês, classificando-as, segundo Bodenheimer referido por Silveira Neto et al. (1976), em constantes onde as espécies estavam presentes em mais de $50 \%$ das coletas, acessórias presentes em $25 \%$ - 50\% e acidentais presentes em menos de 25\%. Também foram utilizados os parâmetros: riqueza $(\mathrm{S})$, abundância $(\mathrm{N})$ e índices de diversidade de Shannon ( $\left.\mathrm{H}^{\prime}\right)$, cujos valores encontrados foram comparados pelo teste " $\mathrm{t}$ " de Student e de Brillouin (H), uniformidade de Shannon (E'), de Brillouin (E) e dominância de Berger-Parker (BP) conforme Magurran (1988) e Krebs (1999), calculados para os meses, períodos mais e, menos chuvoso e total, através do programa de computador "Krebs Ecological Methodology for Windows" (Brzustowski, 1998) e, o percentual de similaridade (PS) entre os meses de coleta (Silveira Neto et al., 1976). As estimativas de riqueza de espécies, para os períodos mais e, menos chuvoso e total, foram realizadas com auxílio do programa de computador "Estimates Richness Estimator" (Colwell, 2009), empregando os procedimentos "Bootstrap", "Chao1", "Chao2", "Jackknife1", Jackknife2" e "MichaelisMentem", utilizando 100 casualizaçôes com abundância de classes igual a 10 (Colwell \& Coddington, 1994). Demais cálculos e gráficos foram realizados através de planilha eletrônica.

\section{RESULTADOS E DISCUSSÃO}

Foram coletados 420 espécimes $(\mathrm{N})$, distribuídas em 64 espécies (S), com representantes de todas as seis tribos da subfamília Arctiinae (Tabela 1), correspondendo a 13,7\% do total conhecido para o Estado e, 19 espécies são novos registros, sendo elas: Agaraea minuta (Schaus, 1892), Amaxia lepida (Schaus, 1912), Calodesma collaris (Drury, 1782), Calodesma dioptis (R. Felder, 1874), Cosmosoma subflamma (Walker, 1854), Cosmosoma stibostictum (Butler, 1876), Cosmosoma thoracica Schaus, 1905, Dinia aeagrus (Cramer, 1779), Disconeura inexpectata (Rothschild, 1910), Dysschema sacrifica (Hübner, [1831]), Dysschema tricolor (Sulzer, 1776), Ennomomima cardytera (Dyar, 1910), Eucereon balium Hampson, 1898, Idalus citrina (Druce, 1890), Isanthrene profusa Hampson,1898, Metaloba argante (Druce, 1897), Robinsonia boliviana Seitz, 1921, Saurita temenus (Stoll, 1781) e Trichromia carinaria (Schaus, 1905).

Das 64 espécies capturadas, $52(81,25 \%)$ ocorreram com menos de dez espécimes e destas, 25 estâo representadas por somente um espécime ("singletons") e 12 por dois ("doubletons"). Cinco espécies ocorreram com mais de 20 espécimes, destacando-se Dycladia lucetius (Stoll, 1781), que foi a mais abundante, com 77 espécimes (Tabela 1). Este padrão de ocorrência de espécies com poucos espécimes parece ser uma tendência para Arctiinae e já encontrado em outros trabalhos (Teston \& Corseuil, 2004, Ferro \& Diniz, 2007; Teston et al., 2009). Isto, também, pode ser verificado em relação à constância (Tabela 1) onde, $67,2 \%$ das espécies foram acidentais (43), 23,4\% acessórias (15) e 9,4\% constantes (6). Das seis constantes, destaca-se $D$. lucetius, pela sua freqüência, a qual ocorreu em todos os meses da amostragem (Tabela 1).

Com relação ao número mensal de espécimes e espécies capturadas neste estudo (Figura 1) observa-se uma variação menor entre os meses durante o período mais chuvoso já, o período menos chuvoso apresentou uma variação maior.



Figura 1 - Números de espécimes e espécies de Arctiinae capturados, mensalmente, com armadilha luminosa em área alterada em Altamira, Amazônia Oriental, Pará, Brasil, para os períodos mais (+ chuva) e menos chuvosos (- chuva), entre dezembro de 2007 a novembro de 2008.

$\mathrm{Na}$ Tabela 2, são apresentados os valores de riqueza de espécies ( $\mathrm{S}$ ), número de espécimes $(\mathrm{N})$, índices de diversidade de Shannon (H') e de Brillouin (H), uniformidade de Shannon (E’) e Brillouin (E) e dominância de Berger-Parker (BP) mensais, por período (mais e menos chuvoso) e total.

Durante o período mais chuvoso obteve-se maior riqueza (S) nos meses de março e abril de 2008, ambas com 15 espécies. A abundância $(\mathrm{N})$ foi maior no mês de março de 2008, com 37 espécimes. Neste mês, o índice de diversidade de Shannon $\left(\mathrm{H}^{\prime}=3,35\right)$ e Brillouin $(\mathrm{H}=2,73)$ foi maior. A uniformidade de Shannon foi maior em maio de 2008 (E' = 0,898), já na de Brillouin o mês de janeiro de 2008 foi a mais alta $(E=0,881)$. Quanto à dominância de Berger-Parker, o mês março apresentou o menor valor $(\mathrm{BP}=0,216)$. Para o período mais chuvoso, a riqueza ( $\mathrm{S}$ ) foi de 41 espécies, abundância (N) 188 espécimes, índice de diversidade de Shannon H' = 4,17, de Brillouin $\mathrm{H}=3,77$, uniformidade de Shannon E' = 
Tabela 1 - Número de espécimes e constância ( $A$ = Acidentais; $B=$ Acessórias; e C = Constantes) de Arctiinae capturados com armadilha luminosa em área alterada em Altamira, Amazônia Oriental, Pará, Brasil, para os períodos mais (+ chuva) e menos chuvosos (- chuva), entre dezembro de 2007 a novembro de 2008.

\begin{tabular}{|c|c|c|c|c|c|c|c|c|c|c|c|c|c|c|}
\hline \multirow{2}{*}{\multicolumn{2}{|c|}{ Tribos / Espécies }} & \multicolumn{6}{|c|}{+ Chuva } & \multicolumn{6}{|c|}{ - Chuva } & \multirow{2}{*}{ Total } \\
\hline & & Dez & Jan & Fev & Mar & Abr & Mai & Jun & Jul & Ago & Set & Out & Nov & \\
\hline \multicolumn{15}{|c|}{ ARCTIINI } \\
\hline 1 & Hypercompe sp. $1^{\text {(A) }}$ & & & & & & & 1 & & & & & & 1 \\
\hline 2 & Paracles laboulbeni (Bar, 1873) (c) & & 1 & 2 & & 1 & & & 3 & 1 & & 1 & 3 & 12 \\
\hline 3 & Paracles sp. $1_{\text {(A) }}$ & & 1 & 1 & & & & & & & & & & 2 \\
\hline 4 & Paracles sp. 2 (c) & 1 & 7 & & 6 & 10 & 2 & 6 & 18 & 1 & & & & 51 \\
\hline 5 & Virbia subapicalis (Walker, 1854) ${ }^{(\mathrm{A})}$ & & & & & & & & & & & 1 & 2 & 3 \\
\hline 6 & Virbia sp. $1^{(\mathrm{A})}$ & & & & & & & & & 3 & & & & 3 \\
\hline \multicolumn{15}{|c|}{ CALLIMORPHINI } \\
\hline & Utetheisa ornatrix (Linnaeus, 1758) ${ }^{(A)}$ & & & & & & & & & 1 & & & & 1 \\
\hline \multicolumn{15}{|c|}{ CTENUCHINI } \\
\hline 8 & Aclytia heber (Cramer, 1780) ${ }^{(\mathrm{A})}$ & & & & & & & & 1 & & & & & 1 \\
\hline 9 & Correbia lycoides (Walker, 1854) ${ }^{(\mathrm{A})}$ & & & & 1 & & & & & & & & & 1 \\
\hline 10 & Correbidia calopteridia (Butler, 1878)(A) & & & & & & & & & & & 1 & & 1 \\
\hline 11 & Correbidia sp. ${ }^{(A)}$ & & & & & & & & & & & & 1 & 1 \\
\hline 12 & Dinia aeagrus (Cramer, 1779) ${ }^{(\mathrm{A})}$ & & & & 1 & & & & 1 & & & & & 2 \\
\hline 13 & Episcepsis lenaeus (Cramer, 1780) ${ }^{(A)}$ & & & & & & & & & & & 1 & & 1 \\
\hline 14 & Eucereon aoris Möschler, 1877 (A) & & 1 & & & & & & & & & & & 1 \\
\hline 15 & Eucereon balium Hampson, 1898 (B) & & & & & & 2 & 1 & 3 & & & & & 6 \\
\hline 16 & Eucereon latifascia (Walker, 1856) (A) & & 1 & & & & & & & & & & & 1 \\
\hline 17 & Eucereon maia Druce, 1884 (A) & 1 & 1 & & & & & & & & & & & 2 \\
\hline 18 & Heliura rhodophila (Walker, 1856) ${ }^{(\mathrm{A})}$ & & 1 & & & & & & & & & 1 & & 2 \\
\hline 19 & Napata leucotela Butler, 1876 (c) & & & 1 & 7 & 1 & & 1 & 2 & & 2 & 2 & & 16 \\
\hline \multicolumn{15}{|c|}{ EUCHROMIINI } \\
\hline 20 & Calonotos triplaga (Hampson, 1909(B) & 15 & & 6 & & & & & & & 4 & 3 & 2 & 30 \\
\hline 21 & $\begin{array}{l}\text { Cosmosoma stibostictum } \\
\text { (Butler, 1876) }^{(\mathrm{A})}\end{array}$ & & & & & & & & & & & 1 & & 1 \\
\hline 22 & $\begin{array}{l}\text { Cosmosoma subflamma } \\
\text { (Walker, 1854) }^{\left({ }^{A}\right)}\end{array}$ & & & & & & 1 & & & & & 2 & & 3 \\
\hline 23 & $\begin{array}{l}\text { Cosmosoma thoracica Schaus, } \\
1905^{(B)}\end{array}$ & 1 & 1 & & 1 & 1 & & 1 & & & & & & 5 \\
\hline 24 & Dycladia lucetius (Stoll, 1781) (c) & 6 & 7 & 13 & 8 & 6 & 4 & 1 & 10 & 8 & 4 & 4 & 6 & 77 \\
\hline 25 & Isanthrene profusa Hampson,1898 ${ }^{(B)}$ & & 1 & & & & & 1 & & 1 & & 1 & 1 & 5 \\
\hline 26 & Leucotmemis torrida (Walker, 1854)(A) & & & & & & & 1 & & & & & & 1 \\
\hline 27 & Leucotmemis sp. ${ }^{(A)}$ & & & 1 & & & & 1 & & & & & & 2 \\
\hline 28 & Macrocneme lades (Cramer, 1776) ${ }^{(A)}$ & & & & & 1 & & & & & & & & 1 \\
\hline 29 & Metaloba argante (Druce, 1897) ${ }^{(\mathrm{A})}$ & 3 & & & & & & & & & & & & 3 \\
\hline 30 & $\begin{array}{l}\text { Phoenicoprocta vacillans } \\
\text { (Walker, 1856) }^{\left({ }^{(A)}\right.}\end{array}$ & 1 & & & & & & & & & & & & 1 \\
\hline 31 & $\begin{array}{l}\text { Poliopastea plumbea } \\
\text { Hampson, } 1898^{(A)}\end{array}$ & & & & & & 1 & & & 1 & & & & 2 \\
\hline 32 & Saurita cassandra (Linnaeus, 1758)(B) & 1 & & 3 & & & 2 & 1 & & 4 & & 1 & & 12 \\
\hline 33 & Saurita temenus (Stoll, 1781) ${ }^{(\mathrm{B})}$ & & & 2 & 1 & & 1 & & 1 & & 2 & & 1 & 8 \\
\hline & Saurita tipulina (Hübner, 1827) ${ }^{(A)}$ & & & & & & & & & & & 1 & & 1 \\
\hline \multicolumn{15}{|c|}{ PERICOPINI } \\
\hline 35 & Calodesma collaris (Drury, 1782) & & & & & & & 4 & 1 & 2 & & & & 7 \\
\hline 36 & Calodesma dioptis (R. Felder, 1874)(A) & & & & & & & 1 & & & & & & 1 \\
\hline 37 & $\begin{array}{l}\text { Dysschema sacrifica (Hübner, } \\
[1831])^{(B)}\end{array}$ & & & & & 1 & 7 & 1 & 8 & & & & & 17 \\
\hline 38 & Dysschema tricolor (Sulzer, 1776) ${ }^{(A)}$ & & & 1 & & & & & & & & & 1 & 2 \\
\hline 39 & Hyalurga leucophlebia Hering, $1925^{(A)}$ & & & & & & & 1 & & & & & & 1 \\
\hline
\end{tabular}




\begin{tabular}{|c|c|c|c|c|c|c|c|c|c|c|c|c|c|c|}
\hline \multirow{2}{*}{\multicolumn{2}{|c|}{ Tribos / Espécies }} & \multicolumn{6}{|c|}{+ Chuva } & \multicolumn{6}{|c|}{ - Chuva } & \multirow{2}{*}{ Total } \\
\hline & & \multirow[t]{2}{*}{ Dez } & \multirow[t]{2}{*}{ Jan } & \multirow[t]{2}{*}{ Fev } & \multirow[t]{2}{*}{ Mar } & \multirow[t]{2}{*}{ Abr } & \multirow[t]{2}{*}{ Mai } & \multirow[t]{2}{*}{ Jun } & \multirow[t]{2}{*}{ Jul } & \multirow[t]{2}{*}{ Ago } & \multirow[t]{2}{*}{ Set } & \multirow[t]{2}{*}{ Out } & \multirow[t]{2}{*}{ Nov } & \\
\hline $\mathrm{PHAl}$ & EGOPTERINI & & & & & & & & & & & & & \\
\hline & Agaraea minuta (Schaus, 1892) ${ }^{\text {(A) }}$ & & & & & & & & & 1 & & & 1 & 2 \\
\hline 41 & Amaxia lepida (Schaus, 1912) ${ }^{(A)}$ & & & & & & & & 1 & & & & & 1 \\
\hline 42 & Azatrephes discalis (Walker, 1856) ${ }^{(A)}$ & & & & & & & & & 1 & & & & 1 \\
\hline 43 & $\begin{array}{l}\text { Disconeura inexpectata } \\
\text { (Rothschild, 1910) }\end{array}$ & & 1 & & 1 & 2 & & 3 & & & & & 1 & 8 \\
\hline 44 & Echeta pandiona (Stoll, [1782]) ${ }^{(A)}$ & & & & & & & & & & & & 1 & 1 \\
\hline 45 & Ennomomima cardytera (Dyar, 1910)(A) & & & & & & 1 & & & & & & & 1 \\
\hline 46 & $\begin{array}{l}\text { Eupseudosoma involuta } \\
(\text { Sepp, [1855]) })^{(A)}\end{array}$ & & & 1 & & & & & & & & 1 & & 2 \\
\hline 47 & Hyperandra novata (Dognin, 1924) & & & & & & & 2 & & & & & & 2 \\
\hline 48 & Idalus admirabilis (Cramer, 1777) ${ }^{(B)}$ & 1 & & & & 2 & & & & 1 & & & & 4 \\
\hline 49 & Idalus citrina (Druce, 1890) ${ }^{(\mathrm{B})}$ & & & & 1 & & 2 & 3 & 1 & 3 & 1 & & & 11 \\
\hline 50 & Idalus critheis Druce, 1884 (A) & & & & 1 & & & & & & & & & 1 \\
\hline 51 & Lepidokirbya vittipes (Walker, 1855) (B) & & & & & 1 & & 1 & & 1 & & & & 3 \\
\hline 52 & Lepidokirbya sp. ${ }^{(A)}$ & & & & 1 & & & & & & & & 1 & 2 \\
\hline 53 & Leucanopsis sp. (B) & 1 & & & & & 1 & 3 & 3 & & & 1 & & 9 \\
\hline 54 & Lophocampa modesta (Kirby, 1892)(A) & & & & 1 & & & & & & & & & 1 \\
\hline 55 & Lophocampa sp. (c) & & & & 1 & 1 & & 3 & 4 & 1 & 1 & 3 & & 14 \\
\hline 56 & $\begin{array}{l}\text { Machaeraptenus ventralis Schaus, } \\
1894 \text { (A) }\end{array}$ & & & & & & & 1 & & 3 & & & & 4 \\
\hline 57 & Melese drucei Rothschild, 1909 (A) & & & & & & & & & 1 & & & & 1 \\
\hline 58 & Melese incertus (Walker, 1855) ${ }^{(\mathrm{C})}$ & & 1 & 2 & 6 & 5 & & 1 & 3 & 4 & & 4 & & 26 \\
\hline 59 & Ormetica sypilus (Cramer, 1777) ${ }^{(\mathrm{B})}$ & & & & & 2 & & & 1 & & & & 1 & 4 \\
\hline 60 & Pareuchaetes aurata (Butler, 1875) ${ }^{(\mathrm{B})}$ & & & 1 & & 1 & 1 & 5 & 16 & 5 & & & & 29 \\
\hline 61 & $\begin{array}{l}\text { Psychophasma erosa (Herrich- } \\
\text { Schäffer, [1858]) }{ }^{(A)}\end{array}$ & & 1 & & & & & & & & & & & 1 \\
\hline 62 & Robinsonia boliviana Seitz, 1921 (A) & & & & & 1 & & & & & & & & 1 \\
\hline & Trichromia carinaria (Schaus, 1905) ${ }^{(\mathrm{A})}$ & & & & & & & & 1 & & & & 1 & 2 \\
\hline & $\begin{array}{l}\text { Trichromia cyclopera } \\
\text { (Hampson, 1905) }^{(\mathrm{A})}\end{array}$ & & & & & & & & 1 & & & & & 1 \\
\hline TOTA & & 31 & 25 & 34 & 37 & 36 & 25 & 44 & 79 & 43 & 14 & 29 & 23 & 420 \\
\hline
\end{tabular}

0,779, de Brillouin E = 0,719 e dominância de Berger-Parker $\mathrm{BP}=0,234$ (Tabela 2).

No período menos chuvoso, a maior riqueza $(S)$ foi encontrada em junho de 2008 (23 espécies), a abundância (N) maior foi em julho de 2008 com 79 espécimes. A diversidade foi maior no mês de junho $\left(\mathrm{H}^{\prime}=4,18\right.$ e $\mathrm{H}=$ 3,37), a uniformidade foi maior em outubro de 2008 ( $\mathrm{E}^{\prime}=$ $0,940$ e $\mathrm{E}=1,01)$, a dominância foi menor em junho $(\mathrm{BP}=$ 0,136). No período menos chuvoso, a riqueza foi 51 espécies, abundância, 232 espécimes, diversidade de Shannon ( $\mathrm{H}^{\prime}=$ $4,70)$, de Brillouin $(\mathrm{H}=4,28)$, uniformidade de Shannon $\left(E^{\prime}=0,829\right)$, de Brillouin $(E=0,771)$ e dominância $\mathrm{BP}=$ 0,142 (Tabela 2).

Os valores do período de menos chuvoso foram superiores para todos os parâmetros analisados, excetuando-se a dominância, onde o valor numérico foi menor e este deve ser inversamente proporcional à diversidade, ou seja, quanto menor a dominância maior a diversidade e uniformidade. Apesar do índice de diversidade de Shannon ser diferente entre os períodos mais e menos chuvoso, estes não apresentaram diferença significativa (Tabela 2).

Com relação ao índice de diversidade de Shannon ( $\left.\mathrm{H}^{\prime}\right)$, não houve diferença significativa nos meses do período mais chuvoso, já no menos chuvoso, o mês de setembro só náo apresentou diferenças com novembro, os demais meses não apresentaram diferenças entre si (Tabela 3). Quando comparamos o índice entre os meses mais e menos chuvoso, o mês de dezembro de 2007 apresentou diferença estatística com os meses de junho, julho, agosto e outubro de 2008 e, fevereiro de 2008 foi diferente de junho do mesmo ano (Tabela 3).

Para o período total diversidade de Shannon foi H’ = 4,69, a de Brillouin, $\mathrm{H}=4,37$, a uniformidade de Shannon foi E' = 
Tabela 2 - Valores de riqueza de espécies (S), número de espécimes (N), índices de diversidade de Shannon $\left(\mathrm{H}^{\prime}\right)^{\star}$ e de Brillouin $(\mathrm{H})^{\star}$, uniformidade de Shannon (E') e Brillouin (E) e dominância de Berger-Parker (BP) mensais e total de Arctiinae capturados com armadilha luminosa em área alterada em Altamira, Amazônia Oriental, Pará, Brasil, para os períodos mais (+ chuva) e menos chuvosos (- chuva), entre dezembro de 2007 a novembro de 2008

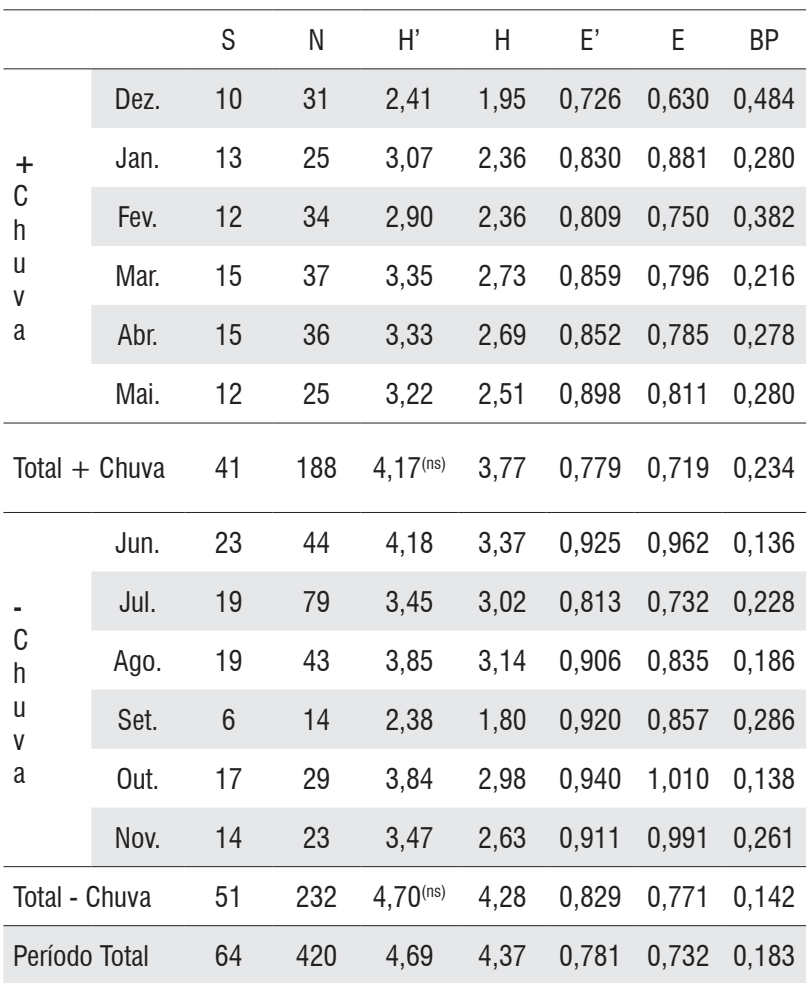

* (Log. - base 2)

"t" não significativo, ao nível de 5\%, na comparação entre período de chuva e seca
0,781, de Brillouin $\mathrm{E}=0,732$ e dominância de Berger-Parker $\mathrm{BP}=0,183$ (Tabela 2).

Quando comparamos a similaridade entre os meses (Tabela 4), notamos que a maior similaridade foi encontrada durante o período mais chuvoso, entre os meses de janeiro e abril de 2008 com $60,8 \%$. E a menor no menos chuvoso, entre os meses de junho e novembro de 2008 com 8,9\% (Tabela 4).

As estimativas de riqueza para os períodos mais e menos chuvoso e total (Tabela 5) mostram que podemos ter um acréscimo de mais espécies na área de estudo.

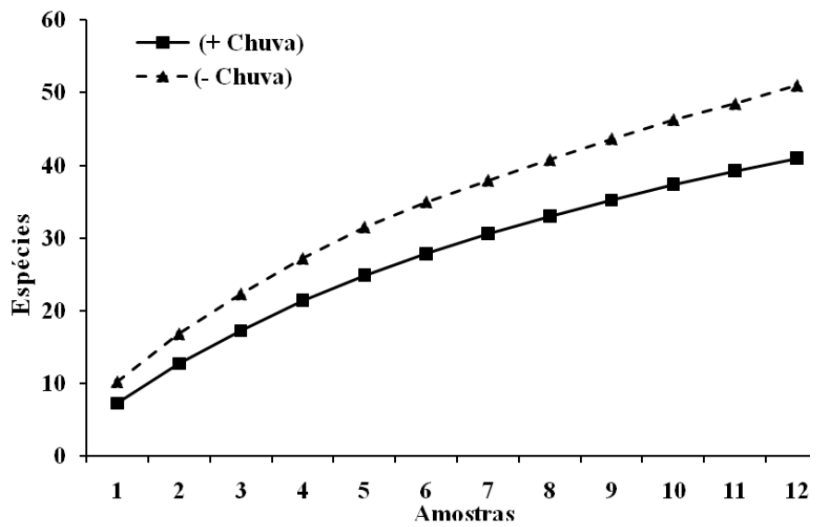

Figura 2 - Curva de acumulação de espécies de Arctiinae capturados com armadilha luminosa em área alterada em Altamira, Amazônia Oriental, Pará, Brasil, para os períodos mais (+ chuva) e menos chuvosos (- chuva), entre dezembro de 2007 a novembro de 2008, em relação ao esforço amostral. Curva construída a partir de dados da riqueza observada média (Sobs).

Tabela 3 - Valores do teste "t" comparando os índices de diversidade de Shannon $\left(H^{\prime}\right)$ mensais para Arctiinae capturados com armadilha luminosa em área alterada em Altamira, Amazônia Oriental, Pará, Brasil, para os períodos mais (+ chuva) e menos chuvosos (- chuva), entre dezembro de 2007 a novembro de 2008.

\begin{tabular}{|c|c|c|c|c|c|c|c|c|c|c|c|c|c|}
\hline & & \multicolumn{6}{|c|}{+ Chuva } & \multicolumn{6}{|c|}{ - Chuva } \\
\hline & & Dez. & Jan. & Fev. & Mar. & Abr. & Mai. & Jun. & Jul. & Ago. & Set. & Out. & Nov. \\
\hline \multirow{4}{*}{$\begin{array}{l}+ \\
\mathrm{C} \\
\mathrm{h} \\
\mathrm{u}\end{array}$} & Dez. & - & & & & & & & & & & & \\
\hline & Jan. & $0,930^{\text {(ns) }}$ & - & & & & & & & & & & \\
\hline & Fev. & $0,774^{\text {(ns) }}$ & $0,234^{\text {(ns) }}$ & - & & & & & & & & & \\
\hline & Mar. & $1,438^{(\mathrm{ns})}$ & $0,375^{\text {(ns) }}$ & $0,667^{\text {(ns) }}$ & - & & & & & & & & \\
\hline \multirow{2}{*}{$\begin{array}{l}\mathrm{v} \\
\mathrm{a}\end{array}$} & Abr. & $1,381^{\text {(ns) }}$ & $0,101^{\text {(ns) }}$ & $0,620^{\text {(ns) }}$ & 0,039 (ns) & - & & & & & & & \\
\hline & Mai. & $1,136^{\text {(ns) }}$ & $0,182^{\text {(ns) }}$ & $0,433^{\text {(ns) }}$ & $0,181^{\text {(ns) }}$ & $0,144^{\text {(ns) }}$ & - & & & & & & \\
\hline \multirow[b]{2}{*}{$C$} & Jun. & $2,499^{(*)}$ & $1,387^{\text {(ns) }}$ & $1,752^{(*)}$ & $1,101^{\text {(ns) }}$ & $1,128^{\text {(ns) }}$ & $1,206^{\text {(ns) }}$ & - & & & & & \\
\hline & Jul. & $1,857^{(*)}$ & $0,565^{\text {(ns) }}$ & $0,936^{\text {(ns) }}$ & $0,158^{\text {(ns) }}$ & $0,200^{\text {(ns) }}$ & $0,348^{\text {(ns) }}$ & $1,090^{\text {(ns) }}$ & - & & & & \\
\hline $\mathrm{h}$ & Ago. & $2,120^{(*)}$ & $1,003^{\text {(ns) }}$ & $1,350^{\text {(ns) }}$ & $0,681^{\text {(ns) }}$ & $0,713^{\text {(ns) }}$ & $0,815^{\text {(ns) }}$ & $0,435^{\text {(ns) }}$ & $0,621^{\text {(ns) }}$ & - & & & \\
\hline u & Set. & $0,047^{\text {(ns) }}$ & $0,908^{\text {(ns) }}$ & $0,755^{\text {(ns) }}$ & $1,370^{\text {(ns) }}$ & $1,320^{\text {(ns) }}$ & $1,100^{\text {(ns) }}$ & $2,372^{(*)}$ & $1,718^{(*)}$ & $2,007^{(*)}$ & - & & \\
\hline \multirow{2}{*}{$\begin{array}{l}\mathrm{v} \\
\mathrm{a}\end{array}$} & Out. & $1,885^{(*)}$ & $0,909^{\text {(ns) }}$ & $1,205^{\text {(ns) }}$ & $0,608^{\text {(ns) }}$ & $0,637^{\text {(ns) }}$ & $0,737^{\text {(ns) }}$ & $0,407^{\text {(ns) }}$ & $0,537^{\text {(ns) }}$ & $0,009^{\text {(ns) }}$ & $1,811^{(*)}$ & - & \\
\hline & Nov. & $1,374^{\text {(ns) }}$ & $0,464^{\text {(ns) }}$ & $0,718^{\text {(ns) }}$ & $0,141^{\text {(ns) }}$ & $0,173^{\text {(ns) }}$ & $0,293^{\text {(ns) }}$ & $0,838^{\text {(ns) }}$ & $0,023^{\text {(ns) }}$ & $0,458^{\text {(ns) }}$ & $1,333^{\text {(ns) }}$ & $0,415^{\text {(ns) }}$ & - \\
\hline
\end{tabular}

(ns) = não significativo ao nível de 5\%; $\left.{ }^{*}\right)$ = significativo ao nível de 5\% 
Tabela 4 - Percentuais de Similaridade (PS) mensais para Arctiinae capturados com armadilha luminosa em área alterada em Altamira, Amazônia Oriental Pará, Brasil, para os períodos mais (+ chuva) e menos chuvosos (- chuva), entre dezembro de 2007 a novembro de 2008.

\begin{tabular}{|c|c|c|c|c|c|c|c|c|c|c|c|c|c|}
\hline & & \multicolumn{6}{|c|}{+ Chuva } & \multicolumn{6}{|c|}{ - Chuva } \\
\hline & & Dez. & Jan. & Fev. & Mar. & Abr. & Mai. & Jun. & Jul. & Ago. & Set. & Out. & Nov. \\
\hline \multirow{4}{*}{$\begin{array}{l}+ \\
\text { C } \\
\text { h } \\
\text { u }\end{array}$} & Dez. & - & & & & & & & & & & & \\
\hline & Jan. & 29,0 & - & & & & & & & & & & \\
\hline & Fev. & 40,2 & 38,9 & - & & & & & & & & & \\
\hline & Mar. & 25,3 & 51,2 & 36,1 & - & & & & & & & & \\
\hline \multirow{2}{*}{$\begin{array}{l}v \\
a\end{array}$} & Abr. & 25,9 & 60,8 & 30,9 & 57,7 & - & & & & & & & \\
\hline & Mai. & 25,7 & 24,0 & 30,9 & 29,4 & 29,6 & - & & & & & & \\
\hline \multirow{2}{*}{$\bar{c}$} & Jun. & 13,3 & 29,0 & 14,3 & 30,8 & 36,0 & 31,9 & - & & & & & \\
\hline & Jul. & 19,1 & 45,6 & 27,0 & 41,7 & 54,1 & 44,9 & 47,8 & - & & & & \\
\hline $\mathrm{h}$ & Ago. & 26,5 & 29,6 & 38,6 & 35,3 & 40,4 & 39,6 & 41,1 & 37,6 & - & & & \\
\hline $\mathrm{u}$ & Set. & 47,9 & 32,0 & 55,0 & 35,1 & 22,2 & 27,1 & 18,2 & 22,8 & 27,9 & - & & \\
\hline \multirow{2}{*}{$\begin{array}{l}v \\
a\end{array}$} & Out. & 30,6 & 32,1 & 42,8 & 35,7 & 35,9 & 24,7 & 21,6 & 30,9 & 33,5 & 38,2 & - & \\
\hline & Nov. & 28,1 & 38,1 & 48,0 & 29,7 & 28,1 & 20,0 & 8,9 & 20,3 & 25,6 & 39,1 & 32,8 & - \\
\hline
\end{tabular}

Tabela 5 - Estimativas para Arctiinae. Número de amostras, espécies, espécies exclusivas, números de espécies representadas por apenas um ("Singletons"), e dois exemplares ("Doubletons") e riqueza de espécies, segundo vários estimadores, capturados com armadilha luminosa em área alterada em Altamira, Amazônia Oriental, Pará, Brasil, para os períodos mais (+ chuva) e menos chuvosos (- chuva), entre dezembro de 2007 a novembro de 2008.

\begin{tabular}{|c|c|c|c|}
\hline & \multicolumn{2}{|r|}{ Período } & \multirow[b]{2}{*}{ Tota } \\
\hline & + Chuva & - Chuva & \\
\hline Amostras & 12 & 12 & 24 \\
\hline Espécies & 41 & 51 & 64 \\
\hline Espécies exclusivas & 13 & 23 & - \\
\hline "Singletons" & 20 & 25 & 26 \\
\hline "Doubletons" & 5 & 6 & 11 \\
\hline "Bootstrap" & 50 & 62 & 76 \\
\hline "Chao 1" & 73 & 94 & 91 \\
\hline "Chao 2" & 60 & 94 & 87 \\
\hline "Jackknife 1" & 60 & 77 & 91 \\
\hline "Jackknife 2" & 71 & 95 & 103 \\
\hline "Michaelis-Mentem" & 70 & 82 & 87 \\
\hline
\end{tabular}

A curva de acumulação de espécies (Figura 2), construída a partir de dados da riqueza observada média (Sobs) para os dois períodos, náo apresentou tendência assíntota. $\mathrm{O}$ comportamento apresentado pelas curvas reflete para um acréscimo de mais espécies, a medida que novos esforços amostrais sejam realizados, o que também foi conferido pelos demais estimadores. O desempenho no período menos chuvoso é devido ao maior número de "singletons e doubletons" (Tabela 5).

\section{CONCLUSÃO}

Constatou-se que $81,25 \%$ das espécies ocorreram com freqüências muito baixas, com até dez espécimes, o que é um padrão para insetos herbívoros em ambientes tropicais e, já encontrado para arctiíneos no Cerrado e em Mata Atlântica no sul do Brasil. O mês de junho de 2008 foi o de maior riqueza e julho o de maior abundância, ambos no período menos chuvoso o que pode ser devido as condiçôes climáticas locais devido a ocorrem poucas variaçóes principalmente pluviométricas, isto influenciando tanto na captura, quanto nas condiçóes durante o desenvolvimento do ciclo de vida dos espécimes.

Dycladia lucetius, foi a espécie mais abundante e a única freqüente em $100 \%$ do período amostrado. Esta espécie possui distribuiçáo muito ampla, sendo provavelmente polífaga e, conseqüentemente, bem adaptada a variaçóes climáticas e a ambientes antrópicos.

No período menos chuvoso foram encontrados os resultados mais significativos para todos os parâmetros analisados, isto pode ser devido as condiçôes microclimáticas mais favoráveis a captura de espécies diferentes, enquanto que no período mais chuvoso foram encontradas as mais significativas percentagens de similaridades entre os meses, onde ocorreram a captura das mesmas espécies em meses diferentes.

Considerando o período total de amostragem há a estimativa de se encontrar entre $18,7 \%$ a $60,9 \%$ de espécies. Sendo que no período menos chuvoso estima-se um acréscimo de $86,3 \%$ a mais de espécies e, no período mais chuvoso $78 \%$. Isto demonstra que mais amostragens devem ser realizadas, inclusive com outras metodologias de captura, pois nenhuma é plenamente eficiente, além do mais, as condiçóes microclimáticas podem afetar a amostragem pontualmente $\mathrm{e}$ desta forma influenciando nas estimativas.

É feito o registro de mais 19 espécies para o Estado destacando que o conhecimento deste grupo é ainda muito pequeno, principalmente em ambientes antrópicos amazônicos. 


\section{AGRADECIMENTOS}

Ao Sr. José Delfino Neto, por liberar a área para o estudo e, também por auxiliar em algumas coletas. A Universidade Federal do Pará pela cota de bolsa PIBIC/UFPA Interior 2007/2008 concedida à segunda autora, acadêmica do Curso de Ciências Biológicas na época do estudo. Ao Prof. Dr. Elio Corseuil, pelo apoio e incentivo nas pesquisas entomológicas. A Patrícia Lazamé Conceição, da Estação de Meteorologia de Altamira (2० DISME - INMET), pelas informaçôes meteorológicas. A Fundação de Amparo à Pesquisas do Estado do Pará, FAPESPA, por conceder apoio por meio do edital Universal 2008, o qual contribuiu na parte final deste.

\section{BIBLIOGRAFIA CITADA}

Brehm, G. 2007. A Contrasting patterns of vertical stratification in two moths families in Costa Rican lowland rain forest. Basic and Applied Ecology, 8(1): 44-54.

Brehm, G.; Axmacher, J.C. 2006. A Comparison of Manual and Automatic Moth Sampling Methods (Lepidoptera: Arctiidae, Geometridae) in a Rain Forest in Costa Rica. Environmental Entomology, 35(3): 757-764.

Brown Jr., K.S.; Freitas, A.V.L. 1999. Lepidoptera. In: Brandão, C.R.F.; Cancello, E.M. (Eds). Biodiversity of the State of Sáo Paulo: synthesis of knowledge at the end of the twentieth century. Volume 5. Invertebrates. FAPESP, São Paulo. p. 225-243 (in Portuguese).

Brzustowski, J. 1998. Krebs Ecological Methodology for Windows, version 0.94. Disponível em: http://www2.biology.ualberta.ca/ jbrzusto/ftp/krebs/index.html. Acesso em 15/06/2009.

Colwell, R.K. 2009. EstimateS: Statistical estimation of species richness and shared species from samples. Version 8.2.0. User's Guide and application. Disponível em: http://viceroy.eeb.uconn.edu/ EstimateS. Acesso em 20/08/2009.

Colwell, R.K.; Coddington, J.A. 1994. Estimating terrestrial biodiversity through extrapolation. Philosophical Transaction of the Royal Society of London, Series B, 345(1311): 101-118.

Conner, W.E. 2009. Tiger Moths and Woolly Bears: Behavior, ecology, and evolution of the Arctiidae. Oxford University Press, New York. 303 pp.

Escada, M.I.S.; Vieira, I.C.G.; Kampel, S.A.; Araújo, R.; Veiga, J.B. da; Aguiar, A.P.D.; Veiga, I.; Oliveira, M.; Pereira, J.L.G.; Carneiro Filho, A.; Fearnside, P.M.; Venturieri, A.; Carriello, F.; Thales, M.; Carneiro, T.S.G.; Monteiro, A.M.V.; Câmara, G. 2005. Occupation processes in the new Amazonian frontiers (the Xingu/Iriri interflow). Estudos Avançados, 19(54): 9-23 (in Portuguese, with abstract in English).

Espírito-Santo, F.D.B.; Oliveira-Filho, A.T.; Machado, E.L.M.; Souza, J.S.; Fontes, M.A.M.L.; Marques, J.J.G.S. 2002. Environmental variables and the distribution of tree species within a remnant of tropical montane semideciduous forest in Lavras, southeastern Brazil. Acta Botanica Brasilica, 16(3): 331356 (in Portuguese, with abstract in English).
Ferro, V.G.; Diniz, I.R. 2007. Composition of the Arctiidae species (Insecta, Lepidoptera) in Cerrado areas. Revista Brasileira de Zoologia, 24(3): 635-646 (in Portuguese, with abstract in English).

Freitas, A.V.L.; Francini, R.B.; Brown Jr., K.S. 2006. Insects as environmental indicators. In: Cullen Jr., L.; Rudran, R.; Valladares-Padua, C. (Orgs). Methods of Study in Conservation Biology and Wildlife Management. 2. Ed. Editora UFPR, Curitiba. p. 125-151 (in Portuguese).

Hawes, J.; Motta, C. da S.; Overal, W.L.; Barlow, J.; Gardner, T.A.; Peres, C.A. 2009. Diversity and composition of amazonian moths in primary, secondary and plantation forest. Journal of Tropical Ecology, 25(3): 281-300.

Heppner, J.B. 1991. Faunal regions and the diversity of Lepidoptera. Tropical Lepidoptera, 2(suppl. 1): 1-85.

Hilt, N.; Brehm, G.; Fiedler, K. 2007. Temporal dynamics of rich moth ensembles in the montane forest zone in southern Ecuador. Biotropica, 39(1): 94-104.

Hilt, N.; Fiedler, K. 2005. Diversity and composition of Arctiidae moth ensembles along a successional gradient in the Ecuadorian Andes. Diversity and Distributions, 11(5): 387-398.

Hilt, N.; Fiedler, K. 2006. Arctiidae moth ensembles along a successional gradient in the Ecuadorian montane rain forest zone: how different are subfamilies and tribes? Journal of Biogeography, 33(1): 108-120.

Hilty, J.; Merenlender, A. 2000. Faunal indicator taxa selection for monitoring ecossystem health. Biological Conservation, 92(2000): 185-197.

Intachat, J.; Woiwod, I.P. 1999. Trap design for monitoring moth biodiversity in tropical rainforests. Bulletin of Entomological Research, 89(2): 153-163.

Kitching, R.L.; Orr, A.G.; Thalib, L.; Mitchell, H.; Hopkins, M. S.; Graham, A.W. 2000. Moths assemblages as indicators of environmental quality in remnants of upland Australian rain forest. Journal of Applied Ecology, 37(2): 284-297.

Kober, E.A.M. 1982. Light trap technical information. EMATER/ RS, Porto Alegre. 24pp (in Portuguese).

Krebs, C.J. 1999. Ecological Methodology. 2a ed. Benjamin/ Cummings, New York. 620 pp.

Landau, B.; Prowell, D.; Carlton, C.E. 1999. Intensive versus longterm sampling to assess lepidopteran diversity in southern mixed mesophytic forest. Annals of the Entomological Society of America, 92(3): 435-441.

Magurran, A.E. 1988. Ecological diversity and its measurement. Princeton University Press, Princeton. 179 pp.

Moraes, B.C. de; Costa, J.M.N. da; Costa, A.L. da; Costa, M.H. 2005. Spatial and temporal variation of precipitation in the State of Pará. Acta Amazonica, 35(2): 207-214 (in Portuguese, with abstract in English).

Salati, E.; Santos, Â.A. dos; Klabin, I. 2006. Relevant environmental issues. Estudos Avançados, 20(56): 107-127 (in Portuguese, with abstract in English). 


\section{ACTA}

Salomão, R. de P.; Vieira, I.C.G.; Suemitsu, C.; Rosa, N. de A.; Almeida, S.S. de; Amaral, D.D. do; Menezes, M.P.M. de. 2007. The forests of Belo Monte on the great curve of the Xingu River, Eastern Amazon. Boletim Museu Paraense Emilio Goeldi. Ciências Naturais, 2(3): 57-153 (in Portuguese, with abstract in English).

Silveira Neto, S.; Nakano, O.; Barbin, D.; Nova, N.A.V. 1976. Manual of Insect Ecology. Agronômica Ceres, Piracicaba, 419 pp (in Portuguese).

Teston, J.A.; Corseuil, E. 2002. Arctiinae (Lepidoptera, Arctiidae) occurent in Rio Grande do Sul State, Brazil. Part I. Pericopini. Biociências, 10(2): 261-268 (in Portuguese, with abstract in English).

Teston, J.A.; Corseuil, E. 2003a. Arctiinae (Lepidoptera, Arctiidae) occurent in Rio Grande do Sul State, Brazil. Part II. Arctiini, Callimorphini e Phaegopterini. Biociências, 11(1): 69-80 (in Portuguese, with abstract in English).

Teston, J.A.; Corseuil, E. 2003b. Arctiinae (Lepidoptera, Arctiidae) occurent in Rio Grande do Sul State, Brazil. Part III. Ctenuchini e Euchromiini. Biociências, 11(1): 81-90 (in Portuguese, with abstract in English).
Teston, J.A.; Corseuil, E. 2004. Diversity of Arctiinae (Lepidoptera, Arctiidae) captured by light traps in six communities in Rio Grande do Sul State, Brazil. Revista Brasileira de Entomologia, 48(1): 77-90 (in Portuguese, with abstract in English).

Teston, J.A.; Specht, A.; Di Mare, R. A.; Corseuil, E. 2006. Arctiinae (Lepidoptera, Arctiidae) collected in protected unities in Rio Grande do Sul State, Brazil. Revista Brasileira de Entomologia, 50(2): 280-286 (in Portuguese, with abstract in English).

Teston, J.A.; Silveira, A.P.; Corseuil, E. 2009. Abundance, Composition and Diversity of Arctiinae (Lepidoptera, Arctiidae) in a Atlantic forest fragment in Iraí, RS, Brazil. Revista Brasileira de Zoociências, 11(1): 65-72 (in Portuguese, with abstract in English).

Recebido em 04/09/2009

Aceito em 08/11/2009 
